\title{
Plasma concentrations of anserine, carnosine and pi-methylhistidine as biomarkers of habitual meat consumption
}

\author{
Patricia Mitry ${ }^{1,2,3} \cdot$ Nina Wawro ${ }^{1,2,3} \cdot$ Sabine Rohrmann ${ }^{4} \cdot$ Pieter Giesbertz $^{5} \cdot$ Hannelore Daniel $^{5} \cdot$ Jakob Linseisen $^{1,2,3,6}$
}

\begin{abstract}
Background/Objectives Dietary intake of red and processed meat has been associated with disease risk. Since dietary intake assessment methods are prone to measurement errors, identifying biomarkers of meat intake in bio-samples could provide more valid intake estimates. We examined associations of habitual red and processed meat, poultry, fish, and dairy products consumption with plasma concentrations of anserine, carnosine, pi-methylhistidine (П-MH), tau-methylhistidine (T-MH), and the ratio of T-MH to $\Pi-\mathrm{MH}$ in a cross-sectional study.

Subjects/Methods Plasma anserine, carnosine, $\Pi-\mathrm{MH}$, and T-MH concentrations were measured using ion-pair LC-MS/MS in 294 participants in the second Bavarian Food Consumption Survey (BVS II). Habitual food consumption was assessed using three 24-h dietary recalls. Associations between plasma metabolites concentrations and meat, fish, eggs, and dairy products consumption were assessed by fitting generalized linear model, adjusted for age, sex, and BMI.

Results Total meat intake was associated with plasma concentrations of anserine, carnosine, $\Pi-\mathrm{MH}$ and, the ratio of T-MH to $\Pi-M H$. Red meat intake was related to carnosine $(p$-trend $=0.0028)$ and $\Pi$-MH plasma levels $(p$-trend $=0.0493)$. Poultry $(p$ trend $=0.0006)$ and chicken $(p$-trend $=0.0003)$ intake were associated with $\Pi-\mathrm{MH}$. The highest anserine concentrations were observed in individuals consuming processed meat or turkey. For T-MH we did not observe any association with meat intake. Conclusions Our results indicate an association between habitual meat consumption and plasma concentrations of anserine, carnosine, $\Pi-\mathrm{MH}$ and the ratio of $\mathrm{T}-\mathrm{MH}$ to $\Pi-\mathrm{MH}$. Intervention studies should clarify whether the analyzed plasma metabolites are indicative for a specific type of meat before proposing them as biomarkers of habitual meat intake in epidemiologic studies.
\end{abstract}

\section{Introduction}

The consumption of total meat, red meat, processed meat, poultry, and fish has been associated with human health and

Jakob Linseisen

j.linseisen@unika-t.de

1 Independent Research Group Clinical Epidemiology, Helmholtz Center Munich, German Research Center for Environmental Health $(\mathrm{GmbH})$, Ingolstaedter Landstr. 1, 85764 Neuherberg, Germany

2 Institute of Epidemiology II, Helmholtz Center Munich, German Research Center for Environmental Health $(\mathrm{GmbH})$, Ingolstaedter Landstr. 1, 85764 Neuherberg, Germany

3 Chair of Epidemiology, Ludwig-Maximilians-Universität disease $[1,2]$. In many populations, meat is an important source of dietary protein and substantially contributes to the supply with several essential nutrients, such as iron or zinc [3]. In the past decade a lot of evidence has been obtained showing that effects on chronic disease risk depend on the type of meat consumed. While a high consumption of red meat and processed meat is associated with a higher risk of, e.g., type-2 diabetes, cardiovascular diseases, some types of

München at UNIKA-T, Neusaesser Str. 47, 86156

Augsburg, Germany

4 Division of Chronic Disease Epidemiology, Epidemiology, Biostatistics and Prevention Institute, University of Zurich, Hirschengraben 84, 8001 Zurich, Switzerland

5 Chair of Nutritional Physiology, Technical University of Munich, Gregor-Mendel-Straße 2, 85354 Freising, Germany

6 ZIEL Institute for Food and Health, Technical University of Munich, Weihenstephaner Berg 1, 85354 Freising, Germany 
cancer, and even total mortality, people consuming predominantly poultry or fish are not at higher risk. In contrast to that a high consumption of fish even decreases the risk of several chronic diseases [4-7].

In observational epidemiological studies, dietary questionnaires such as food frequency questionnaires and dietary recalls are utilized to estimate habitual daily intake of food [8]. However, dietary questionnaires are prone to different types of bias leading to inaccurate estimates of dietary intake [9], resulting in misclassification of participants and impairment of risk estimates of diet-disease relationships [10]. Hence, identifying new biomarkers of meat intake in bio-samples provides the chance to get additional dietary intake information that is not affected by the same measurement error [10-12]. Biomarkers can complement the dietary intake information and help to evaluate critically the available dietary intake data. They can be used to classify a person's dietary intake and in turn will enhance the assessment of the relationship between diet and chronic disease [13]. Ideally, such metabolites can aid in categorizing participants according to the type and amount of meat consumed.

Several studies have analyzed many single compounds in body fluid samples regarding their correlation with meat and fish intake [3, 11]. Carnosine [3], anserine [3], pimethylhistidine (П-MH) [12], tau-methylhistidine (T-MH) [12], and several other metabolic compounds have been suggested as candidate biomarkers of dietary meat intake, as they are found in various amounts in all different types of meat [3]. A recent short-term intervention study confirmed that urinary concentrations of some of the above-mentioned compounds are fairly good biomarkers of meat intake [14]. It remains unclear, however, whether these markers can also be used to characterize an individual's habitual dietary meat intake, the information required in observational (cohort) studies for testing diet-disease associations. Since epidemiological studies usually collect blood samples but often lack (24-h) urinary samples, biomarker measurement in plasma or serum samples can be done in most existing studies.

Thus, in the present study we investigate whether habitual meat, fish, egg, and dairy products consumption is associated with plasma concentrations of anserine, carnosine, П-MH, T-MH or the ratio of $\mathrm{T}-\mathrm{MH}$ to $\Pi-\mathrm{MH}$ in a cross-sectional study. Instead of using the terms 1- and 3methylhistidine we used the terms tau-methylhistidine ( $\mathrm{T}$ $\mathrm{MH}$ ) and pi-methylhistidine (П-MH), respectively, according to $\mathrm{N}^{\mathrm{T}}$ and $\mathrm{N}^{\Pi}$ atoms in the imidazole ring, following the most common IUPAC nomenclature [15] to avoid confusion about the numbering of atoms in the imidazole ring of histidine. The methylhistidine structure derived from anserine metabolism is termed as $\Pi-\mathrm{MH}$, whereas the other methylhistidine structure is named as T-MH.

\section{Methods}

\section{Study participants}

The second Bavarian Food Consumption Survey (BVS II), conducted between September 2002 and June 2003, included 1050 participants aged 13-82 years. The overall participation rate was $70 \%$. The study was designed as a representative cross-sectional study of the German-speaking Bavarian population to describe their dietary and lifestyle habits. In face-to-face interviews, demographics, lifestyle factors, and medical history of the participants were assessed [16]. The BVS II study was approved by the Ethics Committee of the Bavarian Medical Association (Bayerische Landesärztekammer). Written informed consent was obtained from each participant in accordance with institutional requirements and the declaration of Helsinki principles.

\section{Dietary data assessment}

The standardized PC-guided program EPIC-Soft [17, 18] was utilized to assess three 24 -h dietary recalls per subject. Trained study personnel conducted telephone interviews to complete two dietary recalls on weekdays and one recall on a weekend for each participant. Intake data were weighted for weekday and weekend to calculate the average daily food intake. Food items were summarized into 16 food groups and 21 subgroups according to the EPIC-Soft classification scheme [17].

Men and women who completed at least one 24-h dietary recall and were at least 18 years of age, participated in blood sampling and anthropometry assessment in the nearest health office of each participant. Blood samples were stabilized with sodium ethylenediamine tetra-acetic acid (EDTA) (1 g/L), centrifuged and separated from blood cells, as described elsewhere in detail [19]. Plasma aliquots were stored at $-80{ }^{\circ} \mathrm{C}$ until lab analysis.

Potential meat intake biomarkers were measured in 294 plasma samples as only for those participants sufficient plasma volume for the lab analysis was available.

\section{Targeted LC-MS/MS amino acid measurements}

Quantitative amino acid analyses were performed using targeted LC-MS/MS based on the method described by Harder et al. [20]. Briefly, plasma samples (10 $\mu$ l) were dissolved in $500 \mu \mathrm{l}$ ice-cold methanol containing an internal standard mixture of 16 deuterated amino acids, derived from ChromSystems (Gräfelfing, Germany), Cambridge Isotope Laboratories (Tewksbury, MA, USA), and SigmaAldrich (Taufkirchen, Germany). Samples were centrifuged $\left(10 \mathrm{~min}, 10^{\circ} \mathrm{C}, 4000 \times \mathrm{g}\right.$ ) and supernatants containing the extract were evaporated to dryness under nitrogen. In the 
second step, amino acids in the dried samples were derivatized to their butyl esters, as described by Gucciardi et al. [21]. Briefly, a mixture of 95\% n-butanol and 5\% acetylchloride $(\mathrm{v} / \mathrm{v})$ was added to the dried samples. Subsequently, the samples were incubated at $60{ }^{\circ} \mathrm{C}$ for $15 \mathrm{~min}$ at $600 \mathrm{rpm}$ (Eppendorf Thermomixer Comfort; Eppendorf, Hamburg, Germany). The derivatized samples were dried and reconstituted in a $300 \mu \mathrm{l}$ mixture of methanol/water/ formic acid (70/30/0.1\% v/v).

The analysis was performed on a triple quadrupole QTRAP 5500 LC-MS/MS system operating in positive ESI mode (AB Sciex, Framingham, MA) equipped with a 1200 series binary pump (Agilent, Santa Clara, CA) and coupled to an HTC pal autosampler (CTC Analytics, Zwingen, Switzerland). Chromatographic separation was achieved using a Zorbax Eclipse XDB-C18 column (length $150 \mathrm{~mm}$, internal diameter $3.0 \mathrm{~mm}$, particle size $3.5 \mu \mathrm{m}$; Agilent). Eluent A consisted of $0.1 \%$ formic acid, $2.5 \mathrm{mM}$ ammonium acetic acid, and $0.005 \%$ heptafluorobutyric acid in water. Eluent B consisted of $0.1 \%$ formic acid, $2.5 \mathrm{mM}$ ammonium acetic acid, and $0.005 \%$ heptafluorobutyric acid in acetonitrile. Analytes were measured in scheduled multiple reaction monitoring (sMRM). For absolute quantification, a 10-point calibration was performed, using a mixture containing all amino acids in the measurement (A9906 amino acid standards, Sigma-Aldrich, Taufkirchen, Germany). Data analysis was done using Analyst 1.5.1 ${ }^{\circledR}$ software (AB Sciex).

Intra-day and inter-day precisions for П-MH and T-MH were evaluated using ClinCheck $^{\circledR}$ control plasma samples (Recipe, München, Germany). Since these control plasma samples do not contain measurable concentrations of anserine and carnosine, we additionally obtained human plasma samples from individuals collected within a time frame of $24 \mathrm{~h}$ after meat intake, and evaluated the intra-day and inter-day precision for anserine and carnosine in these samples. Except for low levels of anserine, coefficients of variation for the precision were within $20 \%$ for the analytes studied here (Supplementary Table 1). Accuracies were between 77 and 119\% for low levels and between 87 and $104 \%$ for high levels. Except for low levels of anserine, sample peaks showed signal-to-noise values above 9, defined as lower limit of quantitation.

\section{Statistical methods}

From three 24-h dietary recalls a weighted (for weekday and weekend day) mean value for every food group or subgroup was derived representing the usual intake in g/day of the respective item. The following food groups and subgroups were used in the present study: "all meat and meat products", "red meat" (sum of beef, pork, and other rarely consumed fresh meat, as wild, lamb etc.), "beef", "pork", "beef and pork", "poultry" (sum of chicken, turkey, ducks, goose, etc.), "chicken", "turkey", "processed meat", "fish and shellfish", "egg and egg products", "milk and dairy products".

For the descriptive analysis, BMI $\left(\mathrm{kg} / \mathrm{m}^{2}\right)$, age of participants (years) and total energy intake $(\mathrm{kcal} / \mathrm{d})$ were analyzed as continuous variables. Smoking status was described as "smoker", "ex-smoker" and "non-smoker". Socioeconomic status was assessed by household net income, educational level of the one who was interviewed and career position of the principal earner. It was categorized into low, low-medium, medium, medium-high, and high, based on the sum score derived from the single variables. The descriptive analysis of main characteristics of the study population was conducted separately for men and women. We report median and interquartile ranges or absolute numbers and relative frequency as appropriate.

To describe differences of non-normally distributed plasma concentrations of anserine, carnosine, П-MH, T$\mathrm{MH}$, and the ratio of T-MH to П-MH across categories of sex, age, BMI, smoking, social class and physical activity, we report geometric means and $95 \%$ CI instead of arithmetic means and standard deviations. Geometric means were obtained by fitting models with PROC GENMOD using log-transformed plasma concentrations and transforming the estimates derived from the model. For this purpose, BMI was categorized into quartiles and age into three groups ("<40 years", " $40-60$ years", “ $\geq 60$ years”). All bivariate models were adjusted for (continuous) age and sex. When examining BMI, we additionally adjusted for total energy intake. When smoking was examined, (continuous) BMI was added as adjustment variable. Physical activity was categorized as either "active" or "inactive" (no regular sports activity). The $p$-trend was derived based on integer scores assigned to the categories of sex, age, BMI, smoking, social class, and physical activity.

Further, we examined the relationship of plasma concentrations of anserine, carnosine, П-MH, T-MH, and the ratio of T-MH to П-MH with food intake: geometric means for categories of food intake were derived by fitting linear models using log-transformed plasma concentrations. The categorization of the food items was done with respect to the intake data. The food groups "all meat and meat products", "processed meat", and "milk and dairy products" were each grouped in quartiles whereas"red meat", "pork", "beef and pork", "poultry", "fish and shellfish", and "eggs and egg products" were grouped into three groups defined by non-consumers and the consumers divided by their median value. For a small group of food items ("beef", "chicken", and "turkey") only consumers and nonconsumers were discriminated. As adjustment variables, only age, sex, and BMI were included, as for smoking, social class and physical activity no significant associations 
were detected. The $p$-trend was derived based on integer scores assigned to the categories of food intake.

In addition, we performed a non-parametric comparison of the consumption of "all meat and meat products", "milk and dairy products", "fish and shellfish", and "eggs and egg products" across groups defined by the quartiles of all biomarkers. When the Kruskal-Wallis test indicated overall significant differences $(p<0.05)$, Dunn's post-hoc test was subsequently carried out. These analyses were restricted to the consumers of the respective food groups.

All statistical analyses were performed using SAS software, Version 9.3 of the SAS System for Windows (Copyright ( $2002-2010$ SAS Institute Inc.).

\section{Results}

The present study included 101 (34.4\%) men and 193 $(65.7 \%)$ women. The mean age of the participants was 54 years in men and 44 years in women. On average, men had a higher BMI and a higher energy intake compared with women. Further baseline characteristics of the study participants are shown in Table 1 , stratified by sex. Mean intake of milk and dairy products was higher in women $(170 \mathrm{~g} / \mathrm{d})$ compared with men $(106 \mathrm{~g} / \mathrm{d})$, whereas total meat intake in men $(157 \mathrm{~g} / \mathrm{d})$ was much higher as compared with women $(91 \mathrm{~g} / \mathrm{d})$. Descriptive data on all analyzed dietary intake of food groups and subgroups in gram per day are presented in Table 1. Plasma biomarker concentrations range from $0.0771 \mu \mathrm{M}$ for anserine in men and $0.0544 \mu \mathrm{M}$ in women to $15.90 \mu \mathrm{M}$ for $\mathrm{T}-\mathrm{MH}$ in men and $12.33 \mu \mathrm{M}$ women, respectively.

Anserine, П-MH, and the ratio of T-MH to $\Pi-\mathrm{MH}$ plasma concentrations did not differ by sex. In contrast, carnosine and $\mathrm{T}-\mathrm{MH}$ concentrations were higher in men as compared to women (Table 2). Plasma concentrations of anserine, T-MH and П-MH were associated with BMI whereas only $\mathrm{T}-\mathrm{MH}$ was significantly associated with age. Consequently, all following analyses were adjusted for sex, age, and BMI. Smoking, social class and physical activity were not associated with any potential meat intake marker, and thus not further considered.

Plasma anserine, carnosine, and П-MH concentrations significantly increased with higher total meat intake whereas the ratio of T-MH to П-MH plasma concentrations decreased (Table 3). Red meat intake was significantly positively related to plasma concentration of carnosine and $\Pi-\mathrm{MH}$. Beef or pork consumption did not show any significant association, whereas the sum of beef and pork intake significantly affected anserine and carnosine plasma concentrations. With higher processed meat consumption, plasma anserine concentrations significantly increased. Furthermore, $\Pi-\mathrm{MH}$ and the ratio of T-MH to П-MH plasma concentrations differed by intake of
Table 1 Dietary and lifestyle characteristics and plasma biomarker concentrations by sex

\begin{tabular}{|c|c|c|c|c|}
\hline & Male & & Female & \\
\hline$n(\%)$ & 101 & $(34.4)$ & 193 & $(65.7)$ \\
\hline Age (years) & 54 & $(41,63)$ & 44 & $(36,57)$ \\
\hline \multicolumn{5}{|c|}{ Food consumption (g/d) } \\
\hline $\begin{array}{l}\text { Milk and dairy } \\
\text { products }\end{array}$ & 105.8 & $\begin{array}{l}(57.8 \\
195.9)\end{array}$ & 170.1 & $\begin{array}{l}(80.6 \\
289.7)\end{array}$ \\
\hline Milk & 20.6 & $(0,99.3)$ & 57.8 & $\begin{array}{l}(3.7 \\
144.3)\end{array}$ \\
\hline Cheese & 27.3 & $(8.6,48.6)$ & 23.2 & $(9.8,38.9)$ \\
\hline Total meat & 157.4 & $\begin{array}{l}(97.1 \\
223.9)\end{array}$ & 91.1 & $(47,135.6)$ \\
\hline Red meat & 31.7 & $(0,89.2)$ & 25.4 & $(0,55.7)$ \\
\hline Beef & 0 & $(0,0)$ & 0 & $(0,0)$ \\
\hline Pork & 0 & $(0,66.1)$ & 0 & $(0,37.7)$ \\
\hline Beef \& pork & 23.6 & $(0,78)$ & 13.9 & $(0,48.9)$ \\
\hline Poultry & 0 & $(0,15)$ & 0 & $(0,10.5)$ \\
\hline Processed meat & 79.4 & $\begin{array}{l}(40.7 \\
120.7)\end{array}$ & 38.1 & $\begin{array}{l}(14.9 \\
68.3)\end{array}$ \\
\hline Fish \& shellfish, & 0 & $(0,23.8)$ & 0 & $(0,17.8)$ \\
\hline $\begin{array}{l}\text { Eggs \& egg } \\
\text { products }\end{array}$ & 0 & $(0,17.4)$ & 0 & $(0,17.1)$ \\
\hline $\begin{array}{l}\text { Total energy } \\
\text { intake }(\mathrm{kcal} / \mathrm{d})\end{array}$ & 2298 & $\begin{array}{l}(2003, \\
2643)\end{array}$ & 1798 & $\begin{array}{l}(1499, \\
2073)\end{array}$ \\
\hline BMI $\left(\mathrm{kg} / \mathrm{m}^{2}\right)$ & 26.4 & $\begin{array}{l}(24.3 \\
28.8)\end{array}$ & 24.6 & $\begin{array}{l}(22.4 \\
28.3)\end{array}$ \\
\hline \multicolumn{5}{|c|}{ Smoking status, $n(\%)$} \\
\hline Never & 41 & $(40.6)$ & 124 & $(64.6)$ \\
\hline Former & 33 & $(32.7)$ & 38 & $(19.8)$ \\
\hline Current & 27 & $(26.7)$ & 30 & $(15.6)$ \\
\hline \multicolumn{5}{|l|}{ Social class, $n(\%)$} \\
\hline Lower & 10 & $(9.9)$ & 29 & $(15)$ \\
\hline Lower-middle & 23 & $(22.8)$ & 48 & $(24.9)$ \\
\hline Middle & 32 & $(31.7)$ & 63 & $(32.6)$ \\
\hline Upper-middle & 17 & $(16.8)$ & 41 & $(21.2)$ \\
\hline Upper & 19 & $(18.8)$ & 12 & $(6.2)$ \\
\hline \multicolumn{5}{|c|}{ Plasma biomarker concentrations $(\mu \mathrm{M})$} \\
\hline Anserine & 0.0771 & $\begin{array}{l}(0.018 \\
0.295)\end{array}$ & 0.0544 & $\begin{array}{l}(0.014 \\
0.254)\end{array}$ \\
\hline Carnosine & 0.0153 & $\begin{array}{l}(0.011, \\
0.022)\end{array}$ & 0.0103 & $\begin{array}{l}(0.007 \\
0.015)\end{array}$ \\
\hline$\Pi$-MH & 1.940 & $\begin{array}{l}(1.090 \\
4.120)\end{array}$ & 1.540 & $\begin{array}{l}(0.750, \\
3.990)\end{array}$ \\
\hline $\mathrm{T}-\mathrm{MH}$ & 15.90 & $\begin{array}{l}(13.20, \\
18.37)\end{array}$ & 12.33 & $\begin{array}{l}(10.13, \\
15.53)\end{array}$ \\
\hline $\mathrm{T}-\mathrm{MH}$ to $\Pi-\mathrm{MH}$ & 2.165 & $\begin{array}{l}(1.315 \\
2.708)\end{array}$ & 2.230 & $\begin{array}{l}(1.226 \\
2.822)\end{array}$ \\
\hline
\end{tabular}

Values are $n(\%)$ or median (IQR)

total poultry and of chicken. In contrast, consumption of turkey was positively associated with plasma anserine concentrations. The potential meat biomarkers were, in general, 


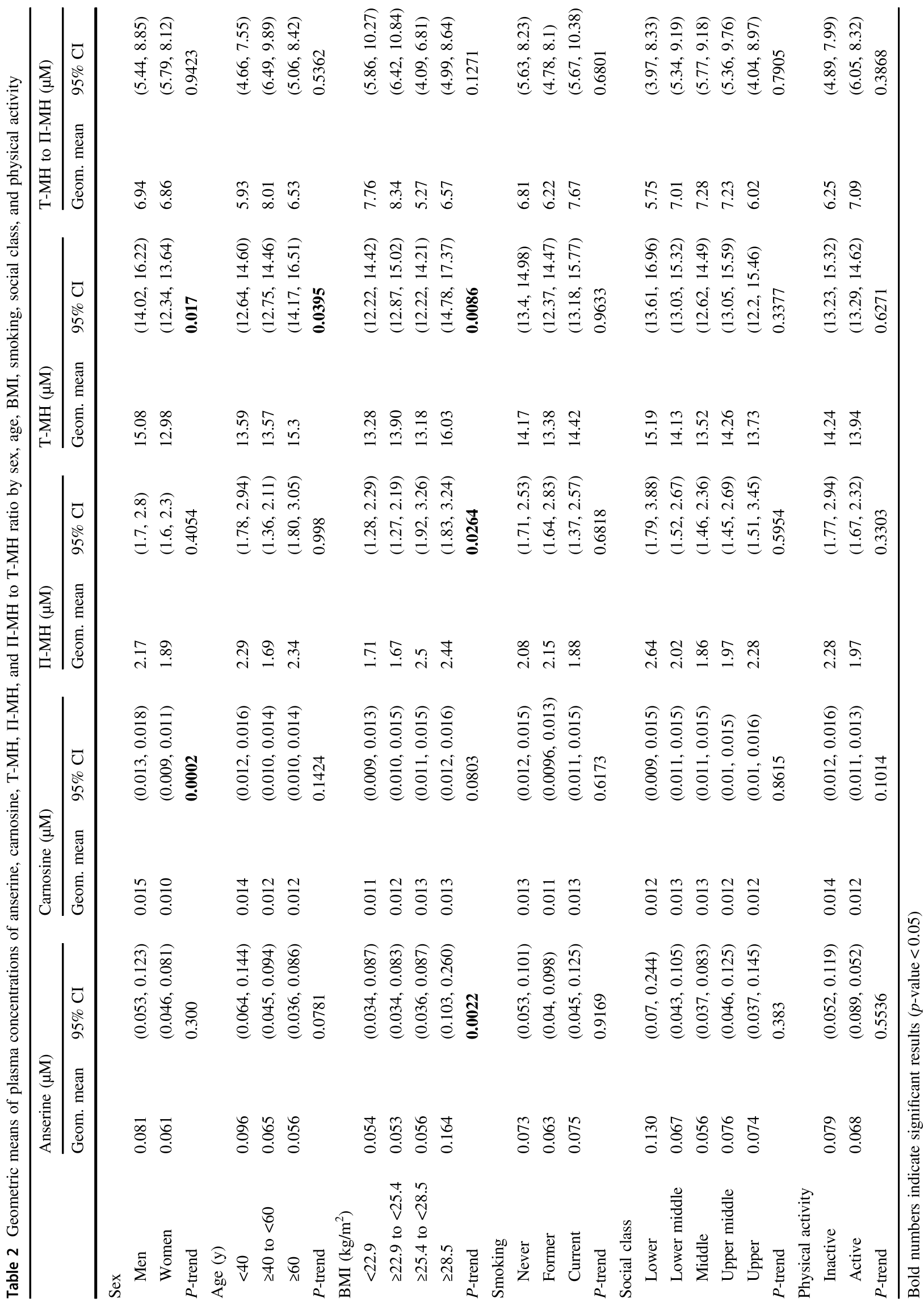




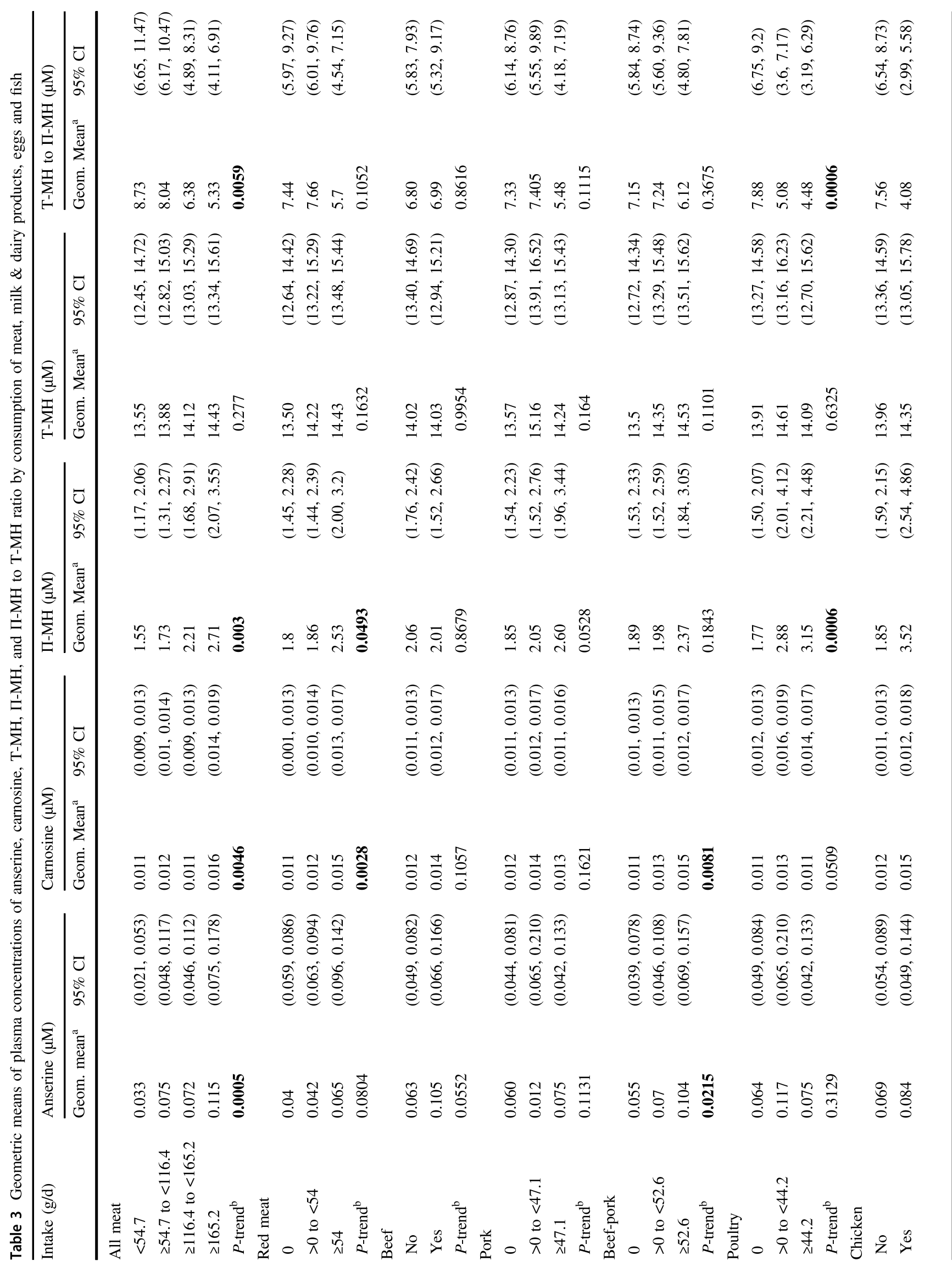




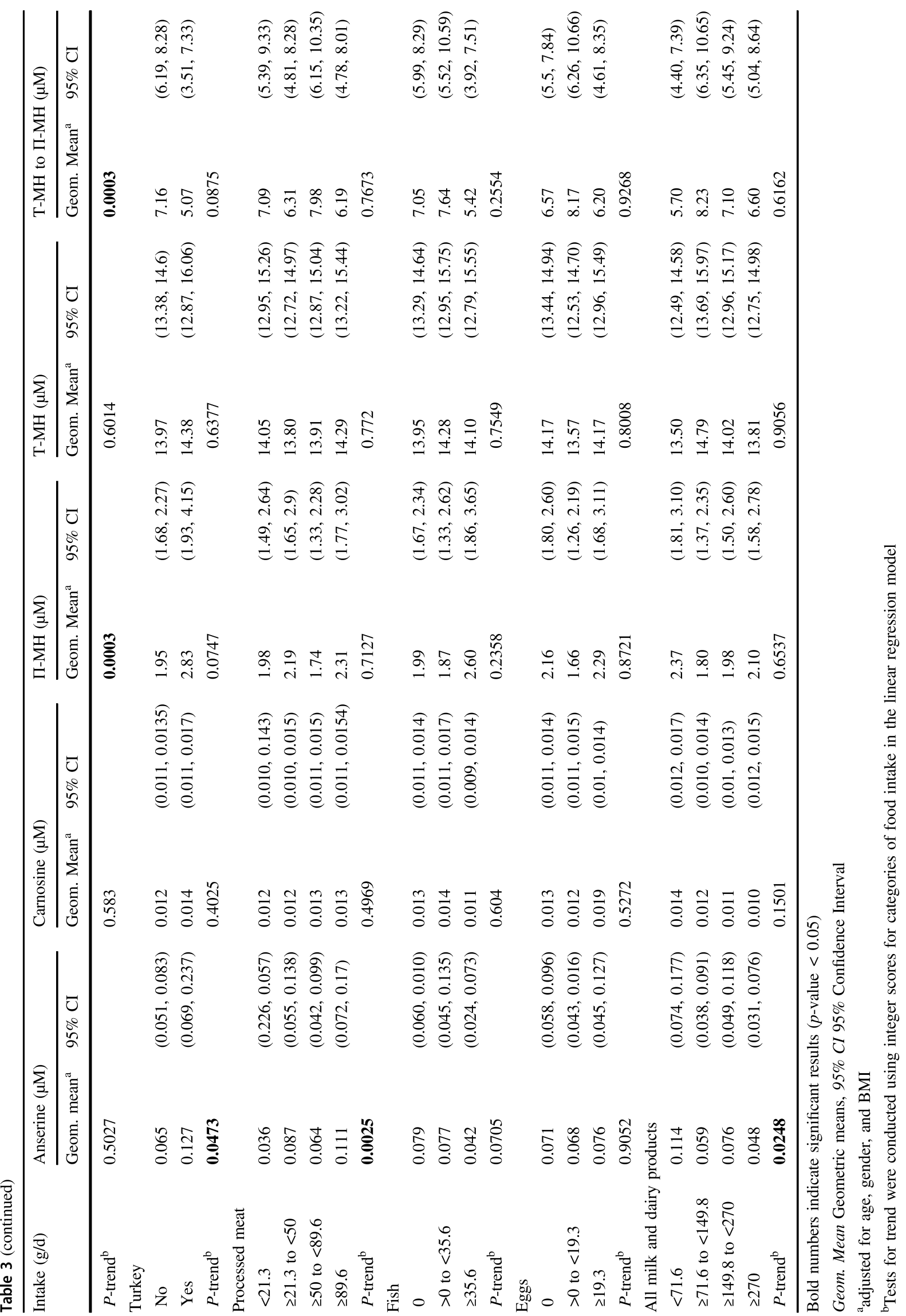


Table 4 Comparison of consumption of all meat, milk and dairy products, fish and shellfish and eggs and egg products by plasma biomarkers (in quartiles, based on all available measurements)

\begin{tabular}{|c|c|c|c|c|c|c|c|c|}
\hline & \multicolumn{2}{|c|}{ All meat } & \multicolumn{2}{|c|}{$\begin{array}{l}\text { Milk and dairy } \\
\text { products }\end{array}$} & \multicolumn{2}{|c|}{ Fish } & \multicolumn{2}{|c|}{ Eggs } \\
\hline & $N$ & Mean rank & $N$ & Mean rank & $N$ & Mean rank & $N$ & Mean rank \\
\hline \multicolumn{9}{|l|}{ Anserine $(\mu \mathrm{M})$} \\
\hline Q1: $<0.0152$ & 72 & $108.22^{\mathrm{a}}$ & 78 & 162.97 & 33 & 48.44 & 32 & 66.81 \\
\hline $\mathrm{Q} 2: \geq 0.0152$ to $<0.0598$ & 70 & $159.64^{\mathrm{b}}$ & 70 & 144.43 & 21 & 47.36 & 30 & 67.12 \\
\hline Q3: $\geq 0.0598$ to $<0.272$ & 72 & $141.78^{\mathrm{a}, \mathrm{b}}$ & 69 & 127.81 & 19 & 53.29 & 36 & 61.24 \\
\hline \multirow[t]{2}{*}{ Q4: $\geq 0.272$} & 72 & $164.81^{\mathrm{b}}$ & 72 & 142.56 & 21 & 40.93 & 29 & 61.10 \\
\hline & & $p=0.0001$ & & $p=0.0861$ & & $p=0.5489$ & & $p=0.8523$ \\
\hline \multicolumn{9}{|l|}{ Carnosine $(\mu \mathrm{M})$} \\
\hline Q1: $<0.008$ & 74 & $104.81^{\mathrm{a}}$ & 78 & $158.03^{\mathrm{a}}$ & 26 & 54.71 & 31 & 65.55 \\
\hline Q2: $\geq 0.008$ to $<0.012$ & 68 & $141.60^{\mathrm{b}}$ & 70 & $154.57^{\mathrm{a}, \mathrm{b}}$ & 22 & 46.05 & 35 & 65.97 \\
\hline Q3: $\geq 0.012$ to $<0.018$ & 75 & $147.88^{\mathrm{b}, \mathrm{c}}$ & 69 & $145.13^{\mathrm{a}, \mathrm{b}}$ & 27 & 39.98 & 30 & 60.07 \\
\hline \multirow[t]{2}{*}{ Q4: $\geq 0.018$} & 69 & $182.11^{\mathrm{c}}$ & 72 & $121.03^{\mathrm{b}}$ & 19 & 50.00 & 31 & 64.03 \\
\hline & & $p<0.0001$ & & $p=\mathbf{0 . 0 3 8 0}$ & & $p=0.2517$ & & $p=0.9189$ \\
\hline \multicolumn{9}{|l|}{ П-МН ( } \\
\hline Q1: $<0.9$ & 71 & $120.37^{\mathrm{a}}$ & 75 & 159.77 & 25 & 41.74 & 30 & 56.30 \\
\hline $\mathrm{Q} 2: \geq 0.9$ to $<1.6$ & 69 & $146.12^{\mathrm{a}, \mathrm{b}}$ & 70 & 141.34 & 22 & 48.00 & 37 & 61.38 \\
\hline Q3: $\geq 1.6$ to $<4.1$ & 73 & $142.12^{\mathrm{a}, \mathrm{b}}$ & 73 & 140.04 & 21 & 50.93 & 32 & 74.73 \\
\hline \multirow[t]{2}{*}{ Q4: $\geq 4.1$} & 73 & $164.90^{\mathrm{b}}$ & 71 & 138.11 & 26 & 49.85 & 28 & 63.45 \\
\hline & & $p=0.0145$ & & $p=0.3595$ & & $p=0.6489$ & & $p=0.2360$ \\
\hline \multicolumn{9}{|l|}{ T-MH $(\mu \mathrm{M})$} \\
\hline Q1: $<10.8$ & 70 & $117.78^{\mathrm{a}}$ & 71 & 153.89 & 19 & 49.55 & 35 & 65.69 \\
\hline Q2: $\geq 10.8$ to $<13.6$ & 69 & $129.33^{\mathrm{a}, \mathrm{c}}$ & 73 & 159.58 & 29 & 42.69 & 29 & 52.55 \\
\hline Q3: $\geq 13.6$ to $<16.9$ & 74 & $154.85^{\mathrm{b}, \mathrm{c}}$ & 73 & 136.36 & 21 & 40.69 & 34 & 69.00 \\
\hline \multirow[t]{2}{*}{ Q4: 1His $\geq 16.9$} & 73 & $170.05^{\mathrm{b}}$ & 72 & 130.21 & 25 & 57.24 & 29 & 67.55 \\
\hline & & $p=0.0005$ & & $p=0.1087$ & & $p=0.1382$ & & $p=0.2847$ \\
\hline \multicolumn{9}{|l|}{ T-MH to П-MH $(\mu \mathrm{M})$} \\
\hline Q1: $<1.248$ & 73 & $163.82^{\mathrm{a}}$ & 71 & 141.89 & 27 & 49.57 & 30 & $60.02^{\mathrm{a}, \mathrm{b}}$ \\
\hline Q2: $\geq 1.248$ to $<2.166$ & 72 & $135.97^{\mathrm{a}, \mathrm{b}}$ & 73 & 141.45 & 22 & 50.68 & 32 & $77.98^{\mathrm{a}}$ \\
\hline Q3: $\geq 2.166$ to $<2.767$ & 72 & $150.90^{\mathrm{a}, \mathrm{b}}$ & 72 & 145.29 & 23 & 44.74 & 38 & $64.97^{\mathrm{a}, \mathrm{b}}$ \\
\hline \multirow[t]{2}{*}{ Q4: $\geq 2.767$} & 69 & $122.14^{\mathrm{b}}$ & 73 & 151.28 & 22 & 44.66 & 27 & $50.48^{\mathrm{b}}$ \\
\hline & & $p=0.0171$ & & $p=0.8866$ & & $p=0.8182$ & & $p=\mathbf{0 . 0 3 3 9}$ \\
\hline
\end{tabular}

Mean ranks and and $p$-value of Kruskal-Wallis test are reported. Letters indicate groups identified by Dunn's post-hoc test $($ alpha $=0.05)$. Only consumers are included in the respective analysis. All group sizes are reported

$Q$ Quartiles

Bold numbers indicate significant results $(p$-value $<0.05)$ not related to the intake of eggs, fish or dairy food. However, there was a significant inverse association between plasma anserine concentrations and dairy food consumption. Such an inverse trend did not reach statistical significance in the case of fish consumption.

The Kruskal-Wallis test revealed significant differences of the consumption of "all meat and meat products" across quartiles for all biomarkers investigated (Table 4 ). The posthoc test showed group differences among the lower and higher quartile groups. The consumption of "milk and dairy products" was only significantly different across carnosine quartiles, whereas consumption of "eggs and egg products" differed only across quartiles of the T-MH to П-MH ratio. These results give reassurance to the findings provided in Table 3.

\section{Discussion}

In the present study, we examined associations of the usual dietary intake and biomarkers measured in plasma. We observed a statistically significant association between total 
meat intake and plasma concentrations of anserine, carnosine, П-MH and the ratio of T-MH to П-MH. Red meat intake was significantly related to carnosine and $\Pi-\mathrm{MH}$ plasma levels. Plasma concentrations of П-MH were significantly related to poultry and chicken intake. The highest mean anserine concentrations were found in individuals consuming processed meat or turkey. For T-MH, we did not observe any association with meat intake.

Red meat, poultry, and fish contain significant amounts of carnosine and its derivatives. Therefore, diet is the main source of carnosine and its derivatives in men [22]. It has been reported $150 \mathrm{~g}$ of beef contains $343.4 \mathrm{mg}$ carnosine and $150 \mathrm{~g}$ of chicken contains $322 \mathrm{mg}$ carnosine [23]; however, information about absorption and bioavailability of histidinedipeptides in human is still inadequate. The dietary histidine-dipeptides carnosine and anserine are rapidly hydrolyzed by carnosinase in plasma and excreted in urine. In total $85-90 \%$ of dietary anserine intake is eliminated as $\Pi$ $\mathrm{MH}$ in urine in human [24]. T-MH is a degradation product of actin and myosin, which are the main proteins found in the striated muscle filaments. T-MH is liberated from these proteins in the gut after meat intake [3] and excreted mainly in urine. T-MH reflects muscle catabolism and muscle mass. A considerable variation in circulating T-MH was observed after consuming the same diet between participants [25]. On the other hand, П-MH is a potential good biomarker for meat intake because it is not affected by muscle mass and activity [26]. To take this potential influence into account, we assessed the bivariate associations of plasma concentrations of our biomarkers with physical activity. No significant associations were found in our study. All analysis were adjusted for BMI, which can at least partly correct for muscle mass, as we found significant associations with our biomarkers investigated. Therefore, we believe that our detected association with meat intake reveals that $\Pi-\mathrm{MH}$ is in fact a marker for meat intake.

Several studies attempted to identify objective biomarkers of meat intake in urine [12, 24, 27, 28] and fewer studies examined plasma or serum samples for meat biomarkers [14, 23, 28-30]. Most studies were intervention studies, e.g., [23, 28, 30] and had comparable analysis methods [23, 28]. To the best of our knowledge, no study has yet examined the association between usual dietary meat intake and plasma carnosine, anserine, T-MH or П$\mathrm{MH}$ concentrations in a cross-sectional study.

Both anserine and carnosine content is highly variable in different types of meat [3]. Both compounds are likely to serve as generic indicators of total meat intake rather than specific quantitative biomarkers as they cannot differentiate between the types of meat that have been consumed. However, some studies suggest that anserine concentrations in urine can be used as a specific biomarker for chicken intake [28, 31]. Yeum et al. [23] analyzed serum anserine concentrations and found anserine serum levels as a marker for chicken intake rather than beef intake. They reported that anserine concentrations in plasma were well detectable, and concluded that the methyl group lowers the affinity of the histidine dipeptides for the carnosinase enzyme, enhancing its blood (serum) stability. However, we did not find any association between chicken intake and anserine plasma level. This could be due to the short elimination half-life of dipeptides [14], as anserine should be a specific biomarker for chicken consumption like П-MH. The reported half-life of anserine is $32 \mathrm{~min}$ [23], though others indicate half-life of $4 \mathrm{~h} \mathrm{[3].} \mathrm{Further,} \mathrm{our} \mathrm{finding} \mathrm{of} \mathrm{an}$ inverse relationship of the consumption of milk and dairy products and fish with anserine concentrations most likely represents a substitution effect, i.e., a lower meat intake in individuals with a high consumption of dairy food or fish.

We did not observe any association between carnosine plasma concentration and beef consumption in contrast to the findings of Park et al. [30]. The peak plasma concentration of carnosine was reached after $2.5 \mathrm{~h}$ of beef consumption, and after $5.5 \mathrm{~h}$ of beef consumption carnosine was not detectable anymore [30]. Since we aimed to capture usual dietary intake habits, we did not measure carnosine plasma concentration directly after beef consumption and therefore, most likely, we missed the carnosine peak. Other reasons that can lead to carnosine disappearance in blood are the rapid uptake of dipeptides into tissues or hydrolysis of carnosine by carnosinase [31]. Because the carnosine plasma concentration depends on the amount of carnosine consumed [30, 31], our sample is likely to show on average low carnosine levels as beef is not regularly consumed in Bavaria, compared with other types of meat [32]. Carnosine has been detected in urine after fish consumption showing an association between carnosine urine concentration and fish intake [24]. However, we did not observe any significant association of carnosine plasma levels and fish consumption.

In line with our results, Yeum et al. [23] reported that carnosine plasma levels were not significantly associated with beef or chicken consumption. They suppose that the carnosine metabolic pathway differs according to the dietary source in humans. For example, beef may have compounds such as carnosine inhibitor or peptides that compete with carnosine in the hydrolytic process, which may stabilize carnosine in serum.

In plasma, П-MH has been found to be the best marker for chicken intake and П-MH levels are increased as well after consumption of red meat and processed meat [28, 33]. This is in good accordance with our findings since we observed additional associations with poultry and all meat food group, although no association with consumption of processed meat was found. Associations of meat and chicken consumptions and П-MH levels have been found in urine as well [25, 28]. П-MH is a short-term biomarker especially for chicken intake rather than describing the 
usual intake [28]. The use of T-MH as a biomarker for meat intake has been investigated in urine as well as in plasma. Cross et al. found a significant increase of T-MH levels in urine after red meat consumption [25], however Dragsted argued that $\mathrm{T}-\mathrm{MH}$ is not a marker of a specific kind of meat [3]. Furthermore, Cheung et al. did not observe any association neither in urine nor in plasma [28]. This is in line with our result of no significant association of dietary intake and $\mathrm{T}-\mathrm{MH}$ plasma levels. One study reported that $1-\mathrm{MH}$ and 3-MH half-lives were 11.7 and $12.6 \mathrm{~h} \mathrm{[12].}$

Urine analyses are less accurate compared to analyses in plasma samples because human muscle catabolism consistently contributes to urinary excretion of T-MH [3]. Fasting T-MH plasma levels are low as after T-MH intake from meat, dipeptides are rapidly excreted in urine [14]. Further, a reason for not obtaining any significant T-MH plasma concentration could be that the frequency of red meat, beef or pork consumption by participants in the BVS II was possibly too low to show a stable association with plasma concentrations of T-MH. The median intake of beef, pork or even poultry was $0 \mathrm{~g} /$ day.

\section{Strength and limitations}

The analytic method used to quantify anserine, carnosine, $\Pi-\mathrm{MH}$ and T-MH in plasma samples is state of the art with a fairly low limit of detection, which has also been applied in other recent studies [23, 28]. Our results are based on 294 plasma samples available of the BVS II study participants who completed three 24-h dietary recalls. Although this is not a random subsample of the BVS II study participants, a differential bias seems unlikely.

Three 24-h dietary recalls are efficient to determine dietary intake at the population level. The use of 24-h dietary recalls is the preferred dietary assessment method, since several studies reported its good relative validity and measurement properties [34-36]. We applied weighting factors to account for consumption differences depending on the day of the week. Yet, some food subgroups such as pork and beef are rarely consumed. For that reason, this study had a limited capacity to assess statistically the particular effect of specific meat subgroups consumption on the related meat intake biomarkers in contrary to the food group of total meat consumption. As we did not assess dietary intake on the day or the day before blood collection, we cannot assess shortterm biomarkers of intake of rarely consumed foods.

\section{Conclusion}

In conclusion, results of this study indicate an association between meat consumption and plasma concentration of anserine, carnosine, П-MH and the ratio of T-MH to П-MH. For epidemiologic studies that did not collect urine samples, these could be valid biomarkers for meat intake. Further investigations of the use of these biomarker combinations as indicators of specific types of meat consumed are needed. In addition, intervention studies should clarify whether the analyzed plasma metabolites are indicative for a specific type of meat.

\section{Data availability}

The datasets generated and/or analyzed during the current study are not publicly available due ethical considerations (informed consent) but are available from the corresponding author on reasonable request.

Acknowledgements The second Bavarian Food Consumption survey (Bayerische Verzehrsstudie II) study was supported by funds of the Bavarian Ministry of Environment, Health and Consumer Protection and the Kurt-Eberhard-Bode-Stiftung. Support for this specific project was provided by The Food Biomarkers Alliance Project FOODBALL (German Ministry for Education and Research, FK 2814ERA02E), a project in the context of the EU Joint Programming Initiative "A Healthy Diet for a Healthy Life".

\section{Compliance with ethical standards}

Conflict of interest The authors declare that they have no conflict of interest.

\section{References}

1. Abete I, Romaguera D, Vieira AR, Lopez de Munain A, Norat T. Association between total, processed, red and white meat consumption and all-cause, CVD and IHD mortality: a meta-analysis of cohort studies. Br J Nutr. 2014;112:762-75. https://doi.org/10. 1017/S000711451400124X

2. Chen GC, Lv DB, Pang Z, Liu QF. Red and processed meat consumption and risk of stroke: a meta-analysis of prospective cohort studies. Eur J Clin Nutr. 2013;67:91-95. https://doi.org/10. 1038/ejen.2012.180

3. Dragsted LO. Biomarkers of meat intake and the application of nutrigenomics. Meat Sci. 2010;84:301-7. https://doi.org/10.1016/ j.meatsci.2009.08.028

4. Micha R, Wallace SK, Mozaffarian D. Red and processed meat consumption and risk of incident coronary heart disease, stroke, and diabetes mellitus: a systematic review and meta-analysis. Circulation. 2010;121:2271-83. https://doi.org/10.1161/ CIRCULATIONAHA.109.924977

5. Chan DS, Lau R, Aune D, Vieira R, Greenwood DC, Kampman E, et al. Red and processed meat and colorectal cancer incidence: meta-analysis of prospective studies. PLoS ONE. 2011;6:e20456 https://doi.org/10.1371/journal.pone.0020456

6. Rohrmann S, Linseisen J. Processed meat: the real villain? Proc Nutr Soc. 2016;75:233-41. https://doi.org/10.1017/S0029665115004255

7. Rohrmann S, Overvad K, Bueno-de-Mesquita HB, Jakobsen MU, Egeberg R, Tjonneland $\mathrm{A}$, et al. Meat consumption and mortality-results from the European Prospective Investigation into Cancer and Nutrition. BMC Med. 2013;11:63 https://doi.org/10. 1186/1741-7015-11-63 
8. Shim JS, Oh K, Kim HC. Dietary assessment methods in epidemiologic studies. Epidemiol Health. 2014;36:e2014009 https:// doi.org/10.4178/epih/e2014009

9. Biro G, Hulshof KF, Ovesen L, Amorim Cruz JA, Group E. Selection of methodology to assess food intake. Eur J Clin Nutr. 2002;56:S25-32. https://doi.org/10.1038/sj.ejcn.1601426

10. Kipnis V, Subar AF, Midthune D, Freedman LS, Ballard-Barbash $\mathrm{R}$, Troiano RP, et al. Structure of dietary measurement error: results of the OPEN biomarker study. Am J Epidemiol. 2003;158:14-21.

11. Turunen AW, Mannisto S, Kiviranta H, Marniemi J, Jula A, Tiittanen $\mathrm{P}$, et al. Dioxins, polychlorinated biphenyls, methyl mercury and omega-3 polyunsaturated fatty acids as biomarkers of fish consumption. Eur J Clin Nutr. 2010;64:313-23. https://doi. org/10.1038/ejen.2009.147

12. Sjolin J, Hjort G, Friman G, Hambraeus L. Urinary excretion of 1methylhistidine: a qualitative indicator of exogenous 3methylhistidine and intake of meats from various sources. Metabolism. 1987;36:1175-84.

13. Potischman N. Biologic and methodologic issues for nutritional biomarkers. J Nutr. 2003;133:875S-80S.

14. Altorf-van der Kuil W, Brink EJ, Boetje M, Siebelink E, Bijlsma $S$, Engberink MF, et al. Identification of biomarkers for intake of protein from meat, dairy products and grains: a controlled dietary intervention study. Br J Nutr. 2013;110:810-22. https://doi.org/ 10.1017/S0007114512005788

15. International Union of Pure and Applied Chemists. IUPAC Gold Book [cited August 2017].

16. Himmerich SGK, Karg G. Bayerische Verzehrsstudie (BVS) IIAbschlussbericht [Second Bavarian Food Consumption SurveyFinal report], Bayerisches Staastministerium fuer Umwelt, Gesundheit und Verbrauchschutz (in German); 2003.

17. Slimani N, Deharveng G, Charrondiere RU, van Kappel AL, Ocke $\mathrm{MC}$, Welch A, et al. Structure of the standardized computerized 24-h diet recall interview used as reference method in the 22 centers participating in the EPIC project. European Prospective Investigation into Cancer and Nutrition. Comput Methods Prog Biomed. 1999;58:251-66.

18. Voss S, Charrondiere UR, Slimani N, Kroke A, Riboli E, Wahrendorf J, et al. EPIC-SOFT a European computer program for 24hour dietary protocols. Z Ernahr. 1998;37:227-33.

19. Himmerich H, Fulda S, Linseisen J, Seiler H, Wolfram G, Himmerich S, et al. TNF-alpha, soluble TNF receptor and interleukin6 plasma levels in the general population. Eur Cytokine Netw. 2006; 17:196-201.

20. Harder U, Koletzko B, Peissner W. Quantification of 22 plasma amino acids combining derivatization and ion-pair LC-MS/MS. J Chromatogr B Anal Technol Biomed Life Sci. 2011;879:495-504. https://doi.org/10.1016/j.jchromb.2011.01.010

21. Gucciardi A, Pirillo P, Di Gangi IM, Naturale M, Giordano G. A rapid UPLC-MS/MS method for simultaneous separation of 48 acylcarnitines in dried blood spots and plasma useful as a secondtier test for expanded newborn screening. Anal Bioanal Chem. 2012;404:741-51. https://doi.org/10.1007/s00216-012-6194-1

22. Gil-Agusti M, Esteve-Romero J, Carda-Broch S. Anserine and carnosine determination in meat samples by pure micellar liquid chromatography. J Chromatogr A. 2008;1189:444-50. https://doi. org/10.1016/j.chroma.2007.11.075
23. Yeum KJ, Orioli M, Regazzoni L, Carini M, Rasmussen $\mathrm{H}$, Russell RM, et al. Profiling histidine dipeptides in plasma and urine after ingesting beef, chicken or chicken broth in humans. Amino Acids. 2010;38:847-58. https://doi.org/10.1007/s00726009-0291-2

24. Abe H, Okuma E, Sekine H, Maeda A, Yoshiue S. Human urinary excretion of L-histidine-related compounds after ingestion of several meats and fish muscle. Int J Biochem. 1993;25:1245-9.

25. Cross AJ, Major JM, Sinha R. Urinary biomarkers of meat consumption. Cancer Epidemiol Biomark Prev. 2011;20:1107-11. https://doi.org/10.1158/1055-9965.EPI-11-0048

26. Datta SP, Harris H. Dietary origin of urinary methylhistidine. Nature. 1951;168:296-7.

27. Myint T, Fraser GE, Lindsted KD, Knutsen SF, Hubbard RW, Bennett HW. Urinary 1-methylhistidine is a marker of meat consumption in Black and in White California Seventh-day Adventists. Am J Epidemiol. 2000;152:752-5.

28. Cheung W, Keski-Rahkonen P, Assi N, Ferrari P, Freisling H, Rinaldi S, et al. A metabolomic study of biomarkers of meat and fish intake. Am J Clin Nutr. 2017;105:600-8. https://doi.org/10. 3945/ajen.116.146639

29. Rohrmann S, Linseisen J, Allenspach M, von Eckardstein A, Muller D. Plasma concentrations of Trimethylamine-N-oxide are directly associated with dairy food consumption and low-grade inflammation in a German Adult Population. $\mathbf{J}$ Nutr. 2016;146:283-9. https://doi.org/10.3945/jn.115.220103

30. Park YJ, Volpe SL, Decker EA. Quantitation of carnosine in humans plasma after dietary consumption of beef. J Agric Food Chem. 2005;53:4736-9. https://doi.org/10.1021/jf047934h

31. Gardner ML, Illingworth KM, Kelleher J, Wood D. Intestinal absorption of the intact peptide carnosine in man, and comparison with intestinal permeability to lactulose. $\mathrm{J}$ Physiol. 1991;439:411-22.

32. Wawro N, Kleiser C, Himmerich S, Gedrich $\mathrm{K}$, Boeing $\mathrm{H}$, Knueppel S, et al. Estimating Usual Intake in the 2nd Bavarian Food Consumption Survey: Comparison of the Results Derived by the National Cancer Institute Method and a Basic Individual Means Approach. Ann Nutr Metab. 2017;71:164-74. https://doi. org/10.1159/000481148

33. Yin X, Gibbons H, Rundle M, Frost G, McNulty BA, Nugent AP et al. Estimation of chicken intake by adults using metabolomicsderived markers. J Nutr. 2017. https://doi.org/10.3945/jn.117.252197

34. Thompson FE, Kirkpatrick SI, Subar AF, Reedy J, Schap TE, Wilson MM, et al. The National Cancer Institute's Dietary Assessment Primer: a resource for diet research. J Acad Nutr Diet. 2015;115:1986-95. https://doi.org/10.1016/j.jand.2015.08.016

35. Slimani N, Bingham S, Runswick S, Ferrari P, Day NE, Welch AA, et al. Group level validation of protein intakes estimated by 24-hour diet recall and dietary questionnaires against 24-hour urinary nitrogen in the European Prospective Investigation into Cancer and Nutrition (EPIC) calibration study. Cancer Epidemiol Biomark Prev. 2003;12:784-95.

36. Crispim SP, de Vries JH, Geelen A, Souverein OW, Hulshof PJ, Lafay L, et al. Two non-consecutive $24 \mathrm{~h}$ recalls using EPIC-Soft software are sufficiently valid for comparing protein and potassium intake between five European centres--results from the European Food Consumption Validation (EFCOVAL) study. Br J Nutr. 2011;105:447-58. https://doi.org/10.1017/S0007114510003648 